\title{
Religion und die Amerikanische Linke: Eine Tradition prägender Begegnungen
}

\author{
Dan McKanan
}

Mehr als zwei Jahrhunderte lang standen religiöse Menschen im Vordergrund der gesellschaftlichen Bewegungen sozialen Wandels in Amerika. Weiße Unitarier, Quäker und die Erweckungsbewegung arbeiteten Seite an Seite mit afroamerikanischen Methodisten und Baptisten im Kampf gegen die Sklaverei. Führende Mitglieder der Arbeiterbewegung des 19. Jahrhunderts schärften ihre Redegewandtheit in Kirchen der Universalisten und in Freidenkergemeinden. Sozialisten proklamierten am Anfang des 20. Jahrhunderts, Jesus sei Sozialist gewesen wie sie. Die hinduistische Lehre Mahatma Gandhis inspirierte die Menschen, die der Rassentrennung im amerikanischen Süden ein Ende machten, ebenso wie Gläubige der römischkatholischen Kirche. Dorothy Day, Gründerin der katholischen Arbeiterbewegung Amerikas, und der katholische Schriftsteller und Mystiker Thomas Merton vermittelten den Gegnern des Vietnamkriegs eine pazifistische Spiritualität. Diese lange Tradition lebt heute in der Occupy-Bewegung weiter, die regelmäßig öffentliche Gottesdienste und private Meditationen abhält. Dasselbe zeigte sich anlässlich der Generalversammlung der Unitarian Universalist Association 2012, während der Tausende Unitarische Universalisten vor einer Haftanstalt für die Rechte von Immigranten ohne Ausweispapiere demonstrierten - und erklärten, ihr religiöser Glaube verpflichte sie, ,auf der Seite der Liebe zu stehen“.

Dieser Beitrag untersucht einige der überraschenden Geschichten aus den Beziehungen zwischen Religion und der amerikanischen Linken. Umstände, die sich allein in den Vereinigten Staaten entwickelten, wie die landesweite Übernahme der 
Religionsfreiheit, machten es für Gläubige einfacher, den sozialen Wandel in den Vereinigten Staaten weiter voranzutreiben als in vielen anderen westlichen Gesellschaften. Als Konsequenz entdeckte die Linke andererseits eine Wahrheit, die auch in jeder anderen Kultur erlebt wird: Wenn Menschen in inniger Begegnung aufeinander treffen, erfahren sie in ihrem Mühen um Freiheit, Gleichheit und Brüderlichkeit einen flüchtigen Blick auf das Göttliche, der ihnen die Kraft gibt, beides zu verändern - sich selbst und ihre Welt.

Ich freue mich sehr über die Möglichkeit, Ihnen etwas über Religion und die Tradition der amerikanischen Radikalen erzählen zu können. Dieses Thema ist resümiert in der Überschrift meines Buches Prophetic Encounters: „Prophetische Begegnungen: Religion und die Tradition der amerikanischen Radikalen".. ${ }^{1}$ Das Buch umfasst zweihundert Jahre Geschichte und erforscht den gegenseitigen Einfluss von Religion und solchen Formen sozialer und politischer Tätigkeiten, die als ,radikal' oder ,links' gelten. Prophetic Encounters untersucht den Sozialismus in seinen beiden Formen, sowohl als utopische als auch als politische Phasen, Arbeiterbewegung, Kampf gegen Sklaverei und um volle politische Rechte für die Nachfahren der Sklaven, die Frauenrechtsbewegung und die Friedensbewegung. Ich behaupte, dass Religion einen wesentlichen Beitrag zu all diesen gesellschaftlichen Bewegungen geliefert hat.

Dieser Vortrag ist für mich die erste Gelegenheit, diesen Teil der Geschichte mit einem europäischen Publikum zu teilen, und ich bin sehr gespannt darauf, Ihre Reaktionen zu hören. Ich vermute, dass einige von Ihnen von der Idee überrascht sein werden, dass Religion ein wichtiger Teil der amerikanischen Linken ist. Sogar in den USA - das ist eine erstaunliche Vorstellung. Weil in Europa Sozialisten und andere linksorientierte Bewegungen eine stärkere Unterstützung erfahren als in den USA, stelle ich mir vor, dass viele Europäer Religion und Sozialismus eher als so etwas wie natürliche Feinde wahrnehmen. Ich verfüge nicht über das Fachwissen, um sagen zu können, ob diese Wahrnehmung tatsächlich für Europa gilt. Aber ich hoffe, Sie überzeugen zu können, dass die Dinge in Amerika nicht so einfach sind. Sehr gerne möchte erfahren, ob Sie meinen, dass mein Beitrag ein neues Licht auf die Geschichte der Linken auch in Europa wirft oder eher nicht.

Ich sollte damit beginnen, mein Argument zu formulieren und die wichtigsten Begriffe zu bestimmen. Meine zentrale Behauptung lautet, dass Religion ein fester Bestandteil aller linksgerichteten Bewegungen in Amerika ist, bis hin zu dem Punkt, dass man die Linke nicht wirklich verstehen kann, ohne die religiöse Dimension zu berücksichtigen. Ich benutze die Ausdrücke, links' und ,amerikanische radikale Tradition', um einen Bezug zu Bewegungen herzustellen, welche die revolutionären Werte von Freiheit, Gleichheit und Brüderlichkeit auf mehr und mehr Personen ausweiten wollen. In Amerika umfassen die wichtigsten dieser Bewegungen die Arbeiterbewegung, Sozialismus, Frauenrechte, Widerstand gegen Sklaverei und gegen Rassismus, die Friedensbewegung. Mit dem Begriff ,Religion' beziehe

${ }^{1}$ Dan McKanan: Prophetic Encounters: Religion and the American Radical Tradition. Boston, Mass. 2011. 
ich mich auf Organisationen, Überzeugungen und Praktiken, die konventionell als solche bezeichnet werden. Das umfasst christliche Gemeinden des amerikanischen mainstream, aber auch Weltreligionen, die von Immigranten nach Amerika gebracht wurden, sowie alternative spirituelle Traditionen wie etwa die Theosophie oder Formen eines Neopaganismus.

Ich behaupte, dass es eine feste Verbindung zwischen der Religion und der Linken gibt - aber ich behaupte nicht, dass diese Verbindung einzigartig ist. Andere soziopolitische Bewegungen mögen ebenfalls intensive Bindungen zur Religion haben. Es ist weithin anerkannt, dass viele der größten und sichtbarsten Organisationen in Amerika sehr konservative soziale Grundsätze vertreten. Diese Gruppen, wie z.B. die Southern Baptist Convention, lehnen die Homosexuellen-Ehe und Abtreibungsrechte ab; in vielen Fällen lehnen sie auch staatliche Sozialhilfe ab, mit der Begründung, dass menschliche Grundbedürfnisse besser über private Spenden finanziert werden. In der Vergangenheit unterstützten einige amerikanische Kirchen den intoleranten Antikommunismus aus der Ära des Kalten Krieges, einige unterstützten die Sklaverei oder mobilisierten Kräfte gegen das Wahlrecht von Frauen. Ich möchte nichts davon herunterspielen. Ebenso wenig möchte ich - wie einige religiöse Linke es taten - vorschlagen, dass eine radikale Religion ,authentischer' religiös sei als eine konservative Religion. Obwohl ich mich persönlich zur Linken bekenne, glaube ich, dass die meisten religiösen Texte und Traditionen zu komplex und mehrdeutig sind, als dass sie das Eigentum einer einzigen Ideologie darstellen könnten.

Wenn ich behaupte, dass es eine feste Verbindung zwischen der Religion und der Linken gibt, verneine ich auch nicht die Existenz von Konflikten zwischen Religion und Radikalismus. Die meisten Führungspersönlichkeiten radikaler Bewegungen in Amerika haben mindestens einige religiöse Organisationen und Ideen scharf kritisiert, und mehr als nur ein paar Radikale haben die Religion als solche verurteilt mit dem Argument, sie stelle das Haupthindernis für eine weitere menschliche Entwicklung dar. Ich habe nicht die Absicht, die Existenz antiklerikaler linker Haltungen zu bestreiten, und ich will sie nicht in jedem Fall verurteilen. Antiklerikale und antireligiöse Haltungen mögen unter manchen Umständen als wohltuend bezeichnet werden. Aber die antiklerikale Einstellung selbst kann nur im Zusammenhang der vielschichtigen Beziehung zwischen der Religion und der Linken verstanden werden.

In Amerika betrachten sich einige der schärfsten Kritiker der MainstreamKirchen als authentischere Christen, als es nach ihrer Ansicht die Mitglieder dieser Kirchen sind. Einige waren Anhänger neuer religiöser Bewegungen, so etwa der Spiritualismus im 19. Jahrhundert oder manche Formen des Neopaganismus im 20. Jahrhundert. Einige dieser Leute waren früher selbst Pfarrer; andere spendeten für sonntägliche Versammlungen, in denen sie mit ihren Anhängern zusammen Lieder singen und Predigten über die Gefahren organisierter Religion hören wollten. 
Es gibt eine feste Beziehung zwischen der Religion und der Linken zum einen einfach deshalb, weil Religion ein fester Bestandteil der menschlichen Erfahrung überhaupt ist. Man kann die Linke nicht verstehen, ohne auch die Religion zu berücksichtigen, weil man die Linke nicht verstehen kann, ohne auch ihre menschliche Komplexität zu berücksichtigen. Ich könnte auch behaupten, Kunst und Literatur seien so sehr Bestandteil der Linken, dass man sie losgelöst von ihren künstlerischen und literarischen Ausdrucksweisen nicht verstehen könne. Doch ich bestehe darauf - in Bezug auf Religion, aber auch auf Kunst und Literatur -, dass Linke und religiöse Bekenntnisse in einer Person nicht einfach nur nebeneinander existieren können, so wie man etwa neben seinem politischen und sozialen Engagement auch Kriminalromane lesen oder den lokalen Sportverein unterstützen mag. Die meisten linken, religiösen und radikalen Bekenntnisse und Erfahrungen sind ineinander verschlungen und miteinander verflochten, jedes prägt und verändert das jeweils andere. Meine Aufgabe im erwähnten Buch war es, die Muster der gegenseitigen Beeinflussung und Veränderung zu erklären.

Mein Buch nannte ich Prophetische Begegnungen, weil mein zentrales Interpretationselement die Begegnung zwischen Menschen ist. Ich glaube, dass alle radikalen Bewegungen mit einer Begegnung beginnen. Wenn Menschen sich auf einer tiefen Ebene begegnen, werden sie dazu inspiriert, zusammenzuarbeiten, um die menschliche Freiheit, Gleichheit und Brüderlichkeit auszuweiten. Solche Begegnungen haben dieselbe Art von Macht wie religiöse Offenbarungen: Sie schenken den Menschen, die sie erfahren, neue Identitäten, neue Wege, die Welt zu verstehen, und die Kraft, einen Wandel in die Welt zu bringen. Religiöse Erfahrungen, in denen es um Begegnungen mit anderen Menschen oder mit Heiligen oder mit übernatürlichen Wesen geht, haben dieselbe Wirkung. Für einige Linke, Religiöse und Radikale verstärken beide Arten von Begegnungen sich untereinander. So war es auch für Dorothy Day, eine Sozialistin in den frühen 1920er Jahren, deren Hingabe an die „Massen“ der Arbeiter in amerikanischen Städten sie dazu brachte, den Glauben der meisten dieser Arbeiter zu ihrem eigenen zu machen: den römischen Katholizismus.

Andere Linke sehen das Linke selbst als die eigentliche und wahre Religion an; konventionelle Religiosität ist im Vergleich dazu nur ein blasser Schatten. So war es bei Dorothy Days Zeitgenossinnen Grace Hutchins und Anna Rochester. Diese Frauen waren protestantische Missionarinnen, die zunächst versuchten, „eine Lösung für unsere drängendsten Probleme“ im „Geist und in der Erfahrung von Jesus Christus“ zu finden, und die dann zu Kommunistinnen wurden. Ihre Konversion zum Kommunismus wurde nicht zuletzt durch eine Literatur angeregt, die den Kommunismus als das wahre Christentum darstellte, die „einen dornenreichen Weg beschritt, um einer weit vor uns liegenden Vision zu folgen.“

Andere Linke wiederum änderten mehrfach die Weise, in der sie die Verbindung zwischen Religion und Radikalismus zu verstehen suchten. A. J. Muste begann seine radikale Karriere als junger protestantischer Pfarrer, der gegen den Ersten Weltkrieg war und deswegen von seiner Kanzel vertrieben wurde. Er setzte 
seine Arbeit als Gewerkschafter fort, gründete nebenbei eine politische marxistische Partei, die den Trotzkisten nahestand. Doch während eines Besuchs in Europa, wo er Leo Trotzki treffen wollte, besuchte er eine Kathedrale, in der er mystische Visionen erfuhr, die ihn zu seinen Wurzeln im christlichen Pazifismus zurückführten. Diese Vision bestimmte seine folgende Laufbahn als „Amerikas erster Pazifist" in den Jahren zwischen dem Ersten Weltkrieg und dem Vietnam-Krieg.

Ich gliedere Prophetische Begegnungen um drei eindeutige Arten der Begegnung herum. Die erste, die ich „Begegnung der Identität“ nenne, findet statt, wenn Menschen ohne institutionalisierte Autorität ihre eigene Macht dadurch entdecken, dass sie zusammenkommen, um Geschichten teilen und für sich selbst eine neue Identität geltend zu machen. In Amerika wird hierbei oft von einer „Bewusstseinserweckung" gesprochen, und sie wird typischerweise mit den radikalen Bewegungen in den 1960er und 1970er Jahren assoziiert, etwa dem Feminismus oder der homosexuellen Emanzipation. Ich habe vergleichbare Praktiken einer „Bewusstseinserweckung" bereits in den 1820ern gefunden, sowohl in der traditionellen Arbeiterbewegung als auch unter afrikanischen Amerikanern, die gegen die Sklaverei kämpften. Ich betrachte die „Begegnung der Identität“, die „Bewusstseinserweckung“, als die Hauptquelle für eine radikale Energie in jeglicher Ära.

Richard Allen und andere afroamerikanische Methodisten machten die Erfahrung einer derartigen Begegnung in der Kirche Saint George in Philadelphia zu Anfang des 19. Jahrhunderts. Als sie zum Gebet niederknieten, zwangen weiße Diakone sie aufzustehen und forderten sie auf, ihr Gebet in einem abgelegenen Außengang zu verrichten. Stattdessen verließen sie die Kirche. Als sie ihre eigene Gemeinde und Glaubensgemeinschaft gründeten, beanspruchten sie eine neue Identität: eine afrikanische. Der zentrale Punkt dieses Anspruches war nicht separatistisch. Die afrikanischen Methodisten wussten, dass auch sie amerikanisch waren. Sie hatten ebenso lange in Nordamerika gelebt wie ihre weißen Nachbarn, und sie hatten genauso hart für die Unabhängigkeit von Großbritannien gekämpft. Aber die Bezeichnung ,afrikanisch' signalisierte die gemeinsamen Wurzeln und eine tiefe Seelenverwandtschaft zwischen den freien Afroamerikanern in Philadelphia und den versklavten Gemeinden des Südens. Sehr bald sprachen sie sich gegen alle Pläne aus, befreite Sklaven nach Afrika zurückzuschicken. „Wir werden uns niemals freiwillig von der versklavten Bevölkerung dieses Landes trennen; sie sind unsere Brüder." Ihr Kampf um eine vollständige Teilhabe an der Gesellschaft Amerikas eröffnete den ein halbes Jahrhundert dauernden Kampf gegen Sklaverei und Rassismus.

Etwa zur gleichen Zeit kamen Arbeiter aus vielen unterschiedlichen Gewerben zusammen, um über die wachsende wirtschaftliche Ungleichheit zu diskutieren. Als einige wenige führende Arbeiter sich mit den kapitalistischen Investoren zusammentaten und Fabriken bauten, sahen die Handwerker ihre eigenen Chancen bedroht. In der Geburtsstunde der amerikanischen Revolution machte dies einen besonders unfairen Eindruck: War es nicht das Ziel der Demokratie, all diesen Leuten mehr Macht zu verleihen? Arbeiter versammelten sich in der Glaubensge- 
meinschaft der Universalisten in Philadelphia, Quäker-Gesellschaften in Wilmington, Methodistenkreise in Baltimore, in Gruppen von Freidenkern an vielen Orten. Genau wie ihre afrikanischen Schwestern und Brüder forderten diese Arbeiter eine neue Identität für sich und eine vollwertige Teilhabe an Amerikas demokratischem Erbe. Der Universalist und Schuhmacher William Heighton ermutigte andere Arbeiter, darüber zu reden, wie sie unter der Übermacht der Reichen ihre demokratisch legitimierte Stimme im politischen Prozess verlören. „Arbeiter“, so erklärte er, ,sollten ausschließlich andere Arbeiter wählen.“ Sie waren die ersten, die Amerikas Wirklichkeit in Begriffen des Klassengedankens deuteten, und sie waren die ersten in der Welt, die klassenbewusste politische Parteien organisierten.

Die Arbeiterpartei revolutionierte die Politik in Philadelphia, New York und anderen Städten für kurze Zeit. Einige ihrer Forderungen, wie kostenlose öffentliche Schulen, wurden von der Mehrheit der anderen Parteien akzeptiert. Aber beide, sie und die afrikanischen Methodisten, hatten nur begrenzten Einfluss, solange sie keine stärker privilegierten Verbündeten hatten. Eine zweite Art „prophetischer Begegnungen" war nun erforderlich, um die radikalen Impulse auf mehr privilegierte Individuen auszuweiten. Diese „persönlichen Begegnungen“ fanden statt, als die Privilegierten auf die neuen Bevollmächtigten trafen, von Angesicht zu Angesicht.

Folgendes geschah, als Frederick Douglass - der entlaufene Sklave, dessen Autobiografien dann zu Klassikern der amerikanischen Literatur wurden - den weiBen Verleger William Lloyd Garrison traf: Als er Douglass seine Geschichte vom Kampf für die Freiheit erzählen hörte, berichtete Garrison, dass er nie zuvor die „gottgleiche Natur“ der Sklavereiopfer so deutlich erkannt hätte. Für Garrison war die Begegnung mit Douglass wie eine Begegnung mit Gott, und er realisierte, dass andere weiße Amerikaner für die Sache der Sklavereigegner gewonnen werden konnten, wenn sie nur Douglass sprechen hören könnten. Das gleiche geschah, als afroamerikanische und weiße Frauen anfingen, Fürbitte für versklavte Personen zu halten, und damit ein starkes kulturelles Tabu im öffentlichen Reden von Frauen brachen. Indem sie es brachen, zwangen sie die Männer dazu, sich auch mit dem vollgültigen Menschsein dieser Frauen auseinanderzusetzen. Diese persönlichen Begegnungen funktionierten, weil das Ablegen eines individuellen Zeugnisses, eines Einspruchs oder Protests bereits protestantische Praxis war, konstitutiv für diese Konfession. Sie ermöglichten die Entwicklung hin zu einer radikalen Theologie, die sich im Wesentlichen auf die Vorstellung stützte, dass jeder Mensch das Bild Gottes spiegelt.

Fast ein Jahrhundert später bezeugte Dorothy Day eine dritte Art prophetischer Begegnungen, als sie den Idealismus beschrieb, der sie zum Sozialismus und zum römischen Katholizismus brachte. Die Sozialisten erblickten im Kollektiv der „Armen und Unterdrückten“ den neuen Messias. Diese Auffassung inspirierte Day, die katholischen Kirchen zu besuchen, die für die armen Einwanderer ihrer Tage die religiöse Heimat waren. Nach ihrer Konversion gründete sie eine Bewegung, die eine Mobilisierung der armen Katholiken anstrebte, um sich für eine 
neue Gesellschaft einzusetzen. Days Hingabe für die gesamte Gemeinde der Armen - nicht nur exemplarisch für einzelne Individuen - war eine Folge der Massengesellschaft, die im Rahmen der Verstädterung und Industrialisierung entstanden war.

Um all die Armen zu treffen, konzipierten und errichteten die neuen Radikalen „Siedlungshäuser" in städtischer Nachbarschaft. Sie entwarfen eine Theologie, die „Sünde“ und „Erlösung“" als gesellschaftliche Begriffe verstand, so dass die Aufstellung einer neuen Gesellschaft mit dem christlichen Versprechen einer Königsherrschaft Gottes gleichgesetzt wurde. Einige strebten den Aufbau einer Art Commonwealth an, die sie einer eschatologischen Königsherrschaft Gottes vorzogen. Wie die persönlichen Begegnungen der Gegner der Sklaverei erhielten die kollektiven Begegnungen ihre Kraft letztlich aus der „Begegnung der Identität“. Die Arbeiter waren bereits dabei, sich in Gewerkschaften selbst zu organisieren, in den Industrial Workers of the World oder in der Sozialistischen Partei.

Die Kraft der Identitätsbegegnungen blieb bis zur Mitte des 20. Jahrhunderts für die meisten Amerikaner der Mittelschicht unbemerkt. Die Dinge änderten sich mit der Erfindung des Fernsehers und dem Aufkommen einer neuen Bewegung, die die Rassentrennung in den Südstaaten beendete. Als afroamerikanische Bewohner von Montgomery (Alabama) einen Boykott organisierten, um die Rassentrennung in städtischen Bussen zu beenden, und als Studenten afroamerikanischer Hochschulen begannen, im ganzen Land auch rassengetrennte Restaurants zu besuchen, waren ihre Aktionen sichtbar für alle Amerikaner. Und alle Amerikaner konnten sehen, wie diese Unterdrückten sich selbst Macht verschafften, indem sie sich zusammentaten. Bald hielten auch andere Gruppen wie Hochschulstudenten, die sich wegen der zunehmenden Militarisierung sorgten, Feministen, Schwule und Lesben, Latinos, amerikanische Indianer, ihre jeweils eigenen bewusstseinserweckenden Treffen ab und gingen über zur politischen Aktion. Diese „Begegnungen der Identität" ermöglichten auch das Entstehen neuer Formen von Religionsgemeinschaften, einschließlich neopaganer Gemeinden, die sich beispielsweise der Anbetung einer Göttin verschrieben, und unterschiedliche Formen liberaler christlicher Theologie.

Diese drei Arten von Begegnungen, jede mit den anderen verbunden, bilden eine einzige, fortgesetzte Tradition des amerikanischen Radikalismus - und nicht nur eine Ansammlung von sich selbst genügenden „Bewegungen“, die auf bestimmte Zeiten und bestimmte Orte beschränkt blieben. In jeder Generation bildeten junge und alte Aktivisten Verbände und wurden inspiriert durch das Gegenüber. Mit anderen Worten: Der amerikanische Radikalismus ist eine Familientradition. Wie in den meisten Familien geht es nicht immer harmonisch zu, und viele der Kämpfe, die auszufechten waren, hatten irgendwie mit Religion zu tun. Freidenker und Marxisten tendieren dazu, die Mainstream-Religion als Teil des Problems zu sehen. Protestanten und Katholiken wiederum betrachten religiöse Institutionen als eine Kraftquelle. 
Aber dieser Wettkampf unter Geschwistern entspringt einer prophetischen Begegnung. All diese „Radikalen“ haben das Göttliche im jeweiligen Gegenüber gesehen, und darum sind ihre Erfahrungen als Radikale religiösen Erfahrungen sehr ähnlich. In radikalen Bewegungen wie in der Kirche bringen Menschen ihre tägliche Routine in Beziehung zu einer größeren geistigen Vision vom Himmel, von Erlösung, von einer neuen Gesellschaft.

Wie es bei Geschwistern eben geschieht, sind konventionelle Religion und Radikalismus oft ungleich. Während Radikale danach streben, auf eine bessere Welt hin zu leben und zu wirken, die es möglicherweise in der Zukunft hier auf der Erde geben könnte, richten viele Gläubige ihr Leben auf eine Realität jenseits dieser Welt aus. Während Radikale erstarkende Identitäten durch Begegnungen mit anderen Menschen erreichen, bieten viele Religionen neue Identitäten durch die Begegnung mit geistigen Wesen an. Einige Radikale finden ihre religiösen und radikalen Bekenntnisse in der gegenseitigen Stärkung; andere gelangen dahin, ihr Anliegen als wahre Kirche zu betrachten.

Diese Unterscheidung zwischen Radikalen, die eine Unterstützung durch die Mainstream-Religion erfahren, und jenen, die den Radikalismus selbst als ihre eigentliche Religion betrachten, bringt mich zurück zu der Frage, ob von dieser amerikanischen Geschichte aus ein verändertes Licht auch auf die europäische Linke fallen kann. Wenn Religiosität mehr oder weniger ein beständiger Aspekt der menschlichen Erfahrung ist, dann muss es eine Art Berührungspunkt zwischen der Religion und dem soziopolitischen Engagement der Linken auch in Europa geben. Aber man wird schwerlich behaupten, dass das europäische Muster dem amerikanischen entspricht. Es kann z.B. sein, dass die Zahl der amerikanischen Linken, die ihr Engagement und die Religion als gegenseitige Kraftquellen betrachten, etwa so groß ist wie derjenigen, die genau das ausschließen - während in Europa die große Mehrheit sie als sich ausschließend betrachtet. Eine solche Unterscheidung zeigt sich offensichtlich etwa in der Beziehung zwischen Immigranten und in Amerika geborenen Sozialisten.

Anfang 1848, als nach der missglückten Revolution Marxisten und andere deutsche Radikale Zuflucht in Amerika suchten, waren sozialistische Immigranten erstaunt über die intensive Religiosität ihrer dort geborenen Verbündeten. Typischerweise organisierten die Immigranten den Parteiapparat, wählten aber als Kandidaten am Ort geborene Pfarrer und andere, die bereit waren, Jesus als einen weltweiten revolutionären Führer zu lobpreisen.

Ich bin sehr daran interessiert, Ihre Meinung darüber zu hören, wie groß die Unterschiede zwischen europäischer und amerikanischer Linker hier sind. Vorausgesetzt, es gibt Unterschiede: Wie lassen sie sich erklären? Ich behaupte, dass der Hauptgrund in der langen amerikanischen Tradition der Religionsfreiheit liegen wird. Die erste Ergänzung zur amerikanischen Verfassung hält fest, dass „der Kongress kein Gesetz bzgl. der Gründung einer Religion schaffen oder eine bestehende freie praktizierte [Religion] verbieten darf". Obwohl dieser Verfassungszusatz zunächst von antiklerikalen Radikalen vorgeschlagen worden war, wurde er 
auch von Mitgliedern minoritärer Religionsgemeinschaften unterstützt, und die meisten Historiker nehmen an, dass dies der Grundstein für das hohe religiöse Engagement in Amerika ist. Der Effekt des First Amendment war die Ersetzung des Monopols ,etablierter ${ }^{6}$ Religionen durch einen gewissermaßen freien religiösen Markt. Religiöse Unternehmer traten Anfang des 19. Jahrhunderts auf und machen bis heute weiter den traditionellen Kirchen Konkurrenz. In diesem Kontext schien es oft eher sinnvoll, seine eigene Religion zu schaffen, als in vorgegebenen Schienen gegen den religiösen Konservatismus anderer Leute zu fahren. Und das ist es, was amerikanische Radikale kontinuierlich getan haben: Sie bauen radikale christliche Gemeinschaften und Gemeinden auf und entwickelten komplett neue religiöse Bewegungen.

Es mag sein, dass auch andere Faktoren einen Beitrag zur unterschiedlichen Ausdifferenzierung von Religion und Radikalismus in Amerika und Europa geleistet haben. Es ist auch durchaus möglich, dass die Unterschiede geringer sind, als sie mir erscheinen, und dass es wichtige Bewegungen unter den europäischen Radikalen gegeben hat, die mit religiösen Traditionen eher kooperativ als kämpferisch umgegangen sind. Ich bin neugierig auf Ihre Ideen. 